\title{
Linx
}

Revue des linguistes de l'université Paris X Nanterre

$10 \mid 1998$

L'indicible et ses marques dans l'énonciation

\section{Temps : entre la langue et le discours}

José Luiz Fiorin

\section{(2) OpenEdition \\ Journals}

Édition électronique

URL : http://journals.openedition.org/linx/977

DOI : $10.4000 /$ linx. 977

ISSN : 2118-9692

\section{Éditeur}

Presses universitaires de Paris Nanterre

\section{Édition imprimée}

Date de publication : 1 juillet 1998

Pagination : 121-148

ISSN : 0246-8743

\section{Référence électronique}

José Luiz Fiorin, «Temps : entre la lanque et le discours », Linx [En ligne], 10 | 1998, mis en ligne le 04 juillet 2012, consulté le 30 avril 2019. URL : http://journals.openedition.org/linx/977 ; DOI : 10.4000/ $\operatorname{lin} x .977$

Ce document a été généré automatiquement le 30 avril 2019.

Département de Sciences du langage, Université Paris Ouest 


\title{
Temps : entre la langue et le discours
}

\author{
José Luiz Fiorin
}

1 Benveniste montre que situer un événement dans le temps chronique est une chose et que l'insérer dans le temps de la langue en est une autre. Pour lui, le temps linguistique est irréductible, soit au temps chronique, soit au temps physique. Ainsi, le linguiste français considère qu'il y a un temps spécifique de la langue $(1974,73)$.

Qu'est-ce qui distingue le temps linguistique des autres notions de temps? «Ce que le temps linguistique a de particulier c'est qu'il est organiquement lié à l'exercice de la parole, qu'il se définit et s'ordonne comme fonction du discours. Ce temps a son centre un centre, à la fois, générateur et axial - dans le présent de l'instance de la parole » (Benveniste, 1974, 73). Le discours instaure un maintenant, moment de l'énonciation. En opposition au maintenant, on crée un alors. Ce maintenant est donc le fondement des oppositions de la langue.

3 Le temps présent indique la contemporanéité de l'événement narré et de la narration. Mais, comme le remarque Benveniste, ce présent, en tant que fonction du discours, ne peut être situé en aucune division particulière du temps chronique, étant donné qu'il les admet toutes, en même temps qu'il n'en exige aucune. En effet, le maintenant est réinventé chaque fois que l'énonciateur énonce, c'est un temps nouveau, encore non vécu, à chaque acte de parole $(1974,74)$.

4 Si le maintenant est généré par l'acte de langage, il se déplace au long du fil du discours tout en demeurant toujours maintenant. Il devient par conséquent un axe qui ordonne la catégorie topologique de la concomitance vs non concomitance. Celle-ci s'articule à son tour en antériorité vs postériorité. De la sorte, tous les temps sont en relation intrinsèque avec l'énonciation. L'axe ordinateur du temps est donc toujours le moment de l'énonciation. Comme le rappelle Benveniste, il semble que ce soit là l'expérience fondamentale du temps dont toutes les langues témoignent à leur manière. Elle informe les systèmes linguistiques particuliers et notamment leur organisation formelle $(1974,75)$.

Comme le maintenant est un temps où un je prend la parole, l'organisation linguistique du temps, comme les autres catégories de l'énonciation, est, pour reprendre une expression 
de Herman Parret $(1988,146)$ égocentrique. Il convient de rappeler, toutefois, que la temporalité de l'énonciateur est acceptée comme la sienne par l'énonciataire. Le maintenant de l'énonciateur est le maintenant de l'énonciataire. La condition d'intelligibilité de la parole réside dans le fait que la temporalité de l'énonciateur, quoique littéralement étrangère et inaccessible à l'énonciataire, est identifiée par celui-ci à la temporalité qui informe sa propre parole lorsqu'il devient à son tour énonciateur. De sorte que le temps du discours n'est ni reporté aux divisions du temps chronique ni refermé sur une subjectivité solipsiste. L'interaction linguistique, qui présuppose un échange intersubjectif, transforme le temps linguistique unipersonnel en omnipersonnel (Benveniste, 1974, 76-77).

6 Le temps linguistique comporte ses propres divisions, en leur ordre propre, indépendamment $\mathrm{du}$ temps chronique. Néanmoins, deux problèmes surgissent. Le premier apparait quand la réception ne se fait pas simultanément à la production (par exemple, une lettre). Dans ce cas, un adverbe comme aujourd'hui n'est plus le signe du présent linguistique au sens restreint, car le lecteur ne peut pas avec rigueur préciser quand il a été proféré, puisque, étant donné qu'on peut le dire de n'importe quel jour du calendrier, on peut l'appliquer à n'importe quel jour indifféremment. Alors, le moyen de rendre intelligibles les marqueurs du temps linguistique c'est de les ancrer dans une division chronique du temps, par exemple, la date. La même chose se produit en ce qui concerne la personne ou l'espace, à savoir, les catégories de l'énonciation ne peuvent être identifiées que par les partenaires de l'échange linguistique. Dans le cas contraire, ils doivent être ancrés en un point déterminé d'un ensemble de coordonnées actantielles ou spatio-temporelles pour pouvoir devenir intelligibles. Dans le cas du temps, l'ancrage se fait à la jonction entre le temps linguistique et le temps chronique (Benveniste, 1974, 77).

D'autre part, la temporalité linguistique est bien démarquée dans ses trois articulations et bien limitée à l'intérieur de chacune d'elles. Prenons l'exemple de l'aujourd'hui. Le temps linguistique centré sur l'aujourd'hui ne peut être déplacé en arrière et en avant que de deux distances-jours : hier et avant-hier et demain et après-demain. Une troisième gradation - avant-avant-hier ou après-après-demain (ou surlendemain) est exceptionnelle; même la seconde distance n'a pas d'expression lexicale indépendante, étant constituée d'hier et demain portés à un degré plus éloigné. Il reste hier et demain, qui sont définis à partir d' aujourd'hui, comme termes originaux pour marquer les distances temporelles à partir du présent linguistique. Quand, pour des raisons pragmatiques, l'énonciateur doit projeter la temporalité au-delà des limites énoncées par hier et demain, le discours sort de son propre plan et utilise la gradation du temps chronique, avec l'énumération des unités : il y a dix jours, dans deux semaines (Benveniste, 1974, 77-78).

8 La temporalité linguistique concerne les relations de successivité entre des états et des transformations représentés dans le texte. Elle ordonne sa progression, montre lesquels sont antérieurs et lesquels sont postérieurs. Ceci implique qu'il y a un système temporel linguistique ordonné par rapport à des repères temporels installés dans le texte, ainsi qu'un système temporel organisé en fonction du présent implicite de l'énonciation. Dans les deux cas, les systèmes temporels servent à organiser la succession d'états et de transformations présents dans le discours. Benveniste $(1976,261-262)$ a remarqué la 
présence de deux systèmes temporels dans la langue. Ils les a appelés systèmes du discours et de l'histoire.

Jusqu'à maintenant, deux points ont été établis pour caractériser la singularité du temps linguistique :

1. son axe ordinateur et générateur est le moment de l'énonciation;

2. il est en relation avec l'ordonnance des états et des transformations narrés dans le texte.

Explicitons un peu mieux cette question. Il existe dans la langue deux systèmes temporels: l'un directement relié au moment de l'énonciation et l'autre ordonné en fonction de moments de référence installés dans l'énoncé. De la sorte, nous avons un système énonciatif dans le premier cas et un système énoncif dans le second. Il se trouve, toutefois, que le moment de référence est en relation avec le moment de l'énonciation, étant donné que celui-ci est l'axe fondamental de l'ordonnance temporelle de la langue. Pour cette raison, au moment de l'énonciation nous appliquons la catégorie topologique concomitance vs non concomitance (antériorité vs postériorité) et nous obtenons trois moments de référence : concomitant, antérieur et postérieur au moment de l'énonciation.

11 Si le moment de référence est concomitant avec le moment de l'énonciation, nous utilisons le système énonciatif, puisque tout se réfère au moment de l'énonciation. Il convient de rappeler que ce moment de référence n'est explicité que dans des cas exceptionnels (comme nous l'avons vu, ceci se produit lorsque la réception et la production ne sont pas simultanées, comme dans le cas d'une lettre, par exemple).

12 Si le moment de référence est antérieur ou postérieur au moment de l'énonciation, ceci doit toujours être explicité. Nous avons donc deux moments de référence explicités : un prétérit et un futur, qui ordonnent deux subsystèmes temporels énoncifs. ${ }^{1}$

13 Le moment des événements (états et transformations) est ordonné par rapport aux différents moments de référence. Cette ordonnance se fait en appliquant la catégorie topologique concomitance vs non-concomitance (antériorité vs postériorité) aux différents moments de référence. Il y a trois moments structurellement remarquables dans la constitution du système temporel : le moment de l'énonciation (ME), le moment de la référence (MR) et le moment de l'événement - ce qui est arrivé (MA).

Nous avons alors, dans la langue, un système temporel énonciatif quand le moment de référence est concomitant avec le moment de l'énonciation, et un système énoncif qui comporte deux subsystèmes : l'un commandé par un moment de référence passé et l'autre par un moment de référence futur. De sorte que, si nous tenons compte, non de la morphologie de la langue, mais des relations constitutives de la catégorie du temps, nous avons neuf temps dans la langue :

1. un système énonciatif (moment de référence concomitant avec le moment de l'énonciation) : concomitance avec le moment de référence - présent ; antériorité à celui-ci passé simple 1 (prétérit parfait); postériorité par rapport au moment de l'énonciation futur simple (futur du présent);

2. un subsystème énoncif du préterit (moment de référence antérieur au moment de l'énonciation) : concomitance limitée par rapport au moment de référence - passé simple 2 (prétérit parfait); concomitance non limitée à celui-ci - prétérit imparfait ; antériorité à ce moment - prétérit plus-que-parfait; postériorité imperfective à ce moment - futur du prétérit simple ; postériorité perfective à ce moment - futur du prétérit composé ; 
3. un subsystème énoncif du futur (moment de référence postérieur au moment de l'énonciation) : concomitance avec le moment de référence - présent du futur ; antériorité à celui-ci - futur antérieur ; postériorité au moment de référence - futur du futur.

Comme ce tableau de temps verbaux ne se fonde pas sur la morphologie, mais sur les relations sémantiques, il peut provoquer un certain étonnement. Pour cette raison, il est nécessaire de faire quelques remarques à ce sujet :

1. Le présent marque une coïncidence entre le moment de l'évènement et le moment de référence présent. Il doit y avoir dans le présent une triple coïncidence $: M A=M R=M E .{ }^{2} \mathrm{Il}$ est nécessaire, néanmoins, de préciser ce qu'est cette coïncidence mentionnée, car le moment de l'énonciation est difficile à délimiter, dans la mesure où il fuit sans cesse. En réalité, le présent est une abstraction de l'esprit, puisque, ainsi que le montre Guillaume, il se recompose avec des instants qui viennent de passer et des instants qui vont encore passer $(1968,51)$. Pour cette raison, la parcelle de temps du moment de référence qui est en relation avec le moment de l'énonciation peut varier en extension. Ainsi, la coïncidence signalée ne doit pas être comprise seulement comme une identité de durée entre deux moments, mais également comme une non-identité entre eux, à condition que le moment de référence, ayant une durée plus longue que le moment de l'énonciation, soit simultané à celui-ci en un point quelconque. Nous pourrions dire que ce qui marque la coïncidence est dans ce cas l'englobement du moment de l'énonciation par le moment de référence. Ce qu'il y a toujours, c'est une coïncidence entre moment de l'événement et moment de référence.

Nous pouvons dénombrer trois cas de relations entre moment de référence et moment de l'énonciation:

a) présent ponctuel : quand il existe une coïncidence entre MR et ME :3

« Un oiseau au plumage bleu raye le tableau en vol diagonal rapide et blesse comme un dard la frondaison de l'acacia » (VGP, 180).

Les verbes soulignés indiquent des événements qui se produisent au moment de référence présent, un maintenant, qui se passe à 6 heures le 18 juin. Comme le moment de référence est un point précis, il y a coïncidence entre celui-ci et le moment de l'énonciation.

b) présent duratif: lorsque le moment de référence est plus long que le moment de l'énonciation. ${ }^{4}$ La durée est variable ; elle peut être courte ou très longue.

1 En outre, elle peut être continue ou discontinue. Lorsqu'elle est discontinue, nous avons le présent itératif ; lorsqu'elle est continue, nous avons le présent dit de continuité :

«Au cours de ce millénaire, l'humanité progresse beaucoup matériellement ».

Le moment de référence est un millénaire et temps de la transformation, progresse, coïncide avec lui.

« Les samedis et dimanches étudiants et professeurs organisent une soirée littéraire et musicale. Et dans le jardin espagnol, ils lisent Racine et Molière sous les étoiles » (VGP, 205).

Le moment de référence (samedis et dimanches) se répète. Le moment de l'événement se répète donc également (organiser et lire). Il y a par conséquent une coïncidence entre eux. Néanmoins, le moment de l'énonciation n'est pas réitéré. Il est unique et ne coïncide qu'en un certain point avec le moment de la référence : dans le présent de l'énonciation la réitération annoncée se produit. Nous avons ici le présent itératif :

"Suivant une habitude américaine, sur le campus étudiants et professeurs

commencent à confraterniser dès le premier jour » (VGP, 205). 
$$
\text { présent progressif. }
$$

«Ele não briga, mas agora está brigando ».

«Il ne se bat pas, mais en ce moment il est en train de se battre ».

31 2. Le prétérit parfait 1 marque une relation d'antériorité entre le moment de l'événement et le moment de référence présent. 
Benveniste avait déjà montré (1974, 238-245) qu'en français, la différence centrale entre le passé composé et le passé simple est que ce dernier est un temps de l'histoire, tandis que l'autre est un temps du discours. Le passé composé indique une antériorité par rapport au présent; le passé simple une concomitance relative à un moment de référence dans le passé. En roumain, en littérature, le passé simple et le passé composé ont les valeurs expliquées ci-dessus (Gramatica Academiei, 1963, I, 239 ; Avram, 1986, 177). En italien, bien que ces temps soient appelés passé lointain et passé proche, terminologie inadéquate, puisqu'on dit due anni fa andammo (passato remoto, c'est-à-dire passé simple) in Scozia. (Il y a deux ans nous sommes allés en Écosse) et Dio ha creato il mondo (Dieu a crée le monde, en employant le passé composé) (Serianni, 1989, 471-473), l'usage est identique à celui du français et du roumain. En portugais, par contre, le prétérit parfait simple conserve toute sa vitalité, parce que le temps composé correspondant n'a pas à proprement parler de fonction temporelle, mais une fonction aspectuelle. En effet, si je dis Jean, ce mois-ci, a lu très tard, a lu situe le début de l'événement dans un moment antérieur au moment de référence présent, et indique en même temps sa continuité dans le moment présent. De cette manière, il a une valeur aspectuelle durative (continuative ou itérative) et inachevée. C'est pourquoi le prétérit parfait simple cumule en portugais deux fonctions: l'antériorité par rapport à un moment de référence présent et la concomitance par rapport à un moment de référence passé. Nous avons donc, du point de vue fonctionnel deux prétérits parfaits : le $\mathrm{n}^{\mathrm{0}} 1$, qui est le temps du système énonciatif, et le $\mathrm{n}^{\circ} 2$, qui appartient au système énoncif. Le passé composé ne conserve sa valeur d'antériorité que dans des cas très restreints, pour exprimer un fait qui vient de se produire. Par exemple, un orateur termine son discours en disant J'ai dit.

3. Le futur du présent indique une postériorité du moment de l'événement par rapport à un moment de référence présent :

«Pergunto-lhes agora que pretendem fazer no segundo semestre de 1944. Alguns permanecerão na Universidade para terminar o curso » (VGP, 198).

«Je leur demande maintenant ce qu'ils prétendent faire au second semestre 1944. Quelques uns resteront à l'Université pour terminer le cours » (VGP, 198).

Le moment de référence maintenant est le 20 juin 1944. Par rapport à ce moment resteront indique une postériorité du moment de l'événement.

On est accoutumé de dire qu'il existe une opposition passé/futur qui permet d'évoquer l'avenir sous l'éclairage du passé.

«Ela foi muito gentil comigo, serei eternamente grato a ela ».

" Elle $a$ été très gentille avec moi, je lui en serai éternellement reconnaissant ».

En réalité, il n'y a pas dans ce cas d'opposition passé/futur, mais une opposition présent/ futur. En effet, l'énoncé au passé implique une énonciation au présent du type j'affirme qu'elle a été très gentille avec moi. Cette énonciation présupposée constitue le moment de référence, et c'est par rapport à ce dernier qu'on utilise le futur du présent.

Cette valeur temporelle du futur détermine, qu'à moins que la proposition exprime une vérité intemporelle, elle ne peut exprimer une modalité factuelle, car sa vraie valeur ne peut être déterminée au moment de l'énonciation. Par conséquent, l'unique possibilité de faire des assertions au futur dépend de l'évaluation faite par l'énonciateur de la nécessité, la probabilité, la possibilité ou l'impossibilité que se produise un certain état de choses. Le futur, en portugais, indique un effet dont la réalisation dépend de ce qu'une certaine cause se vérifie (Si l'inflation n'est pas subjuguée, il y aura une explosion sociale), signale que la 
phrase où il apparait est le contenu propositionnel d'un acte illocutoire commissif (Je jure que ce crime ne restera pas impuni); montre l'état de choses décrit comme contingent (Avec cette circulation, nous arriverons en retard). Le futur indique également que l'on considère nécessaire, impossible ou hautement probable la réalisation d'un événement donné dans un moment postérieur au présent, quoique dans ces cas le portugais préfère le présent de l'indicatif. La non-factualité des événements exprimés par le futur fait qu'ils ont toujours une valeur modale accouplée à leur valeur temporelle (Mira Mateus et alii, 1983, 118-123). Normalement, on dit que le futur peut occuper la place de l'impératif dans l'expression d'un ordre. Dans le décalogue, nous avons par exemple: Tu ne tueras point. Cette valeur dérive de la portée temporelle et non modale du futur. Comme l'ordre porte sur des événements postérieurs par rapport au moment présent, le futur peut se substituer à l'impératif.

41 4. La concomitance du moment de l'événement par rapport à un moment de référence passé peut être exprimée aussi bien par le prétérit parfait 2 que par le prétérit imparfait. Il est donc nécessaire d'établir un autre axe pour distinguer la valeur de ces deux temps verbaux. La différence entre eux réside dans le fait que chacun d'eux possède une valeur aspectuelle distincte: le prétérit parfait 2 signale un aspect limité, et par conséquent accompli, dynamique, tandis que le prétérit imparfait marque un aspect non limité, et par conséquent, inachevé, statique. Pour cette raison Bakhtin, acceptant les thèses de Lorck sur ces deux temps, dit qu'avec le parfait « notre regard se tourne vers l'extérieur, vers le monde des objets et des contenus que la pensée a déjà appris » (et que pour cette raison il considère accomplis); avec l'imparfait « il se tourne vers l'intérieur, vers le monde des pensées en devenir et en procès de constitution » (et pour ce motif il voit les états et les transformations indiqués par ce temps, en cours au long d'un espace de temps (1979, 170-171).

42 Si nous prenons deux phrases telles que: "Le 29 décembre, le Sénat condamna le Président Collor à la peine de huit ans d'incapacité politique » et «Le 29 décembre, le Sénat condamnait le Président Collor à la peine de huit ans d'incapacité politique », nous constatons que condamna aussi bien que condamnait indiquent la concomitance par rapport à un moment de référence passé (29 décembre 1992). Cependant, dans le premier cas, on considère l'action comme quelque chose d'achevé, comme une discontinuité (un point) dans la continuité du moment de référence, et donc comme quelque chose de dynamique, vu de l'extérieur; dans le second, l'action est considérée comme inachevée, continue dans la continuité du moment de référence, comme quelque chose de statique, vu de l'intérieur, durant son déroulement.

43 Comme l'imparfait a une valeur durative et que la durativité peut être continue ou discontinue (itérativité), l'imparfait peut aussi bien exprimer un fait qui se répète dans le passé qu'un fait continu dans le passé (ce qu'on appelle l'imparfait descriptif) :

«Quando a criança via o sol irisando a água do lago, batia as mãos de contentamento ».

«Quand l'enfant voyait le soleil irisant l'eau du lac, il battait des mains de contentement».

Dans ce cas, le moment de référence passé qui est implicite, est «toutes les fois qu'il y avait du soleil ».

«Sete horas da manhã haviam de ser. A luz de um sol esplêndido fluía no éter que a trovoada da véspera tinha acendrado » $(\mathrm{T}, 11)$. 
«Il devait être sept heures du matin. La lumière d'un soleil splendide se répandait dans l'éther que l'orage de la veille avait purifié » $(T, 11)$. relation, son aspect est toujours perfectif. Il y a, en portugais, deux formes de ce temps verbal : une forme simple et une forme composée.

« Está terminado o terrível ano de 1992. Sob a regência do presidente da República, uma quadrilha assumira o controle da máquina do Estado » (VEJA, 30/12/1992, 1268, 32).

«L'Année terrible 1992 est achevée. Sous la conduite du président de la République, une bande de malfaiteurs avait pris en mains le contrôle de la machine de l'État ». parfait avait pris en mains (assumira) indique que le fait s'est produit avant le moment de référence.

"Quand nous nous sommes séparés, elle avait l'apparence classique d'une dame, avec une jupe et une blouse qui faisaient trop sérieux. Elle avait cessé d'être séduisante » (VEJA, 10/10/1995, 62).

51 Par rapport au moment de référence passé quand nous nous sommes séparés, avait cessé exprime une antériorité.

doit se rappeler que la forme analytique se substitue peu à peu à la forme synthétique dans la langue parlée. Ceci tient au fait que l'opposition latine infectum vs perfectum a été refaite dans les langues romanes sous des formes composées parallèles à celles des temps de l'infectum (sauf dans le cas du prétérit parfait $1 \mathrm{du}$ portugais - passé simple - pour les raisons déjà expliquées). De sorte que, suivant une tendance des langues romanes, la forme analytique du prétérit plus-que-parfait a la possibilité d'exprimer à la fois la relation d'antériorité et l'aspect perfectif, tandis que la forme synthétique ne présente que la relation d'antériorité. Comme au plus-que-parfait les deux significations sont associées, il est naturel que la forme synthétique soit de moins en moins utilisée. 

par rapport au moment de référence passé.

"ACM disse que Collor seria inapelavelmente derrotado no Senado" (VEJA, 30/12/1992, 1268, 21).

«ACM a dit que Collor serait vaincu sans appel au Sénat ».

Le moment de référence passé est l'instant où $\mathrm{ACM}$ a dit quelque chose à quelqu'un. La défaite est un fait qui aura lieu à un moment postérieur au moment de référence. C'est pourquoi il est exprimé au futur du prétérit.

Le futur du prétérit a la plupart du temps le caractère d'une anticipation imaginaire. Si le futur du présent n'exprime pas une modalité factuelle mais surgit comme une expectative, le futur du passé (le conditionnel) a une valeur hypothétique.

La forme composée marque, de même que la forme simple, un fait postérieur par rapport à un moment de référence passé. Cependant, elle indique un fait antérieur à un autre événement dans l'avenir. En d'autres termes, pour l'emploi du futur du prétérit composé (passé du conditionnel $1^{\text {re }}$ forme) on tient compte de deux moments de référence : il est postérieur à l'un d'eux et antérieur à l'autre. Pour cette raison, on pourrait l'appeler futur du prétérit du prétérit. La distinction entre les deux formes du futur du prétérit pourrait être considérée comme aspectuelle: la forme simple est imperfective et la forme composée est perfective. Il est évident que ces aspects doivent être considérés dans leur rapport avec la perspective temporelle où ces temps se situent.

«Todos supunham que, quando o inverno chegasse, a guerra na Bósnia teria terminado.»

Tout le monde supposait que, lorsque l'hiver arriverait, la guerre en Bosnie serait finie ».

« Ontem de manhã, eu sabia que, às dez horas, o avião já teria chegado ».

«Hier matin, je savais qu’à dix heures l'avion serait déjà arrivé ».

Dans la première phrase, le moment de référence passé est le moment de la supposition. Par rapport à lui, l'arrivée de l'hiver est postérieure. Par rapport à l'arrivée de l'hiver, la fin de la guerre en Bosnie est antérieure. Dans la seconde phrase, le moment de référence passé est hier matin. Le moment dix-heures lui est postérieur, tandis que l'arrivée de l'avion aura lieu avant dix heures.

7. Le présent du futur n'as pas en portugais de forme spécifique; il est exprimé par un futur du présent simple ou par un futur du présent progressif (futur du présent de l'auxiliaire être (estar en portugais) + le gérondif) corrélatifs à un futur du présent du subjonctif explicite ou implicite (par exemple, « Au moment où je te donnerai un signal, tu lanceras les fusées ») (No momento em que eu lhe der um sinal, você soltará os rojões).

59 8. L'antériorité par rapport à un moment de référence futur est indiquée par le futur antérieur qui, dans la nomenclature grammaticale brésilienne est appelé futur du présent composé (par exemple, « Quand vous arriverez, j'aurai terminé le travail »).

9. La postériorité par rapport à un moment de référence futur (futur du futur) est indiquée par le futur du présent simple en corrélation avec un autre futur du présent simple (le futur simple); l'ultériorité de l'un par rapport à l'autre sera marquée, de manière explicite ou implicite, par le mot après, ou un parasynonyme ( Tu prendras ton bain et ensuite tu pourras regarder la télévision »).

Les adverbes et les locutions adverbiales de temps s'articulent également en un système énonciatif et énoncif. Le premier est centré sur un moment de référence présent, identique au moment de l'énonciation; le second est organisé autour d'un moment de 
référence (passé ou futur) inscrit dans l'énoncé, ce qui signifie en ce qui concerne les adverbes qu'il n'existe pas un subsystème en relation avec un moment de référence passé et un autre lié à un moment de référence futur. À chacun des moments de référence (énonciatif et énoncif) s'applique la catégorie topologique concomitance vs non concomitance (antériorité vs postériorité). Prenons un seul exemple. Pour exprimer les termes de la catégorie topologique, nous avons dans le système énonciatif aujourd'hui, hier et demain. Dans le système énoncif leur correspondent ce jour-là, la veille, le lendemain. Les locutions avec prochain appartiennent au système énonciatif; celles avec suivant au système énoncif. Les parlants les confondent souvent. Voyons un exemple :

«Mais la décision de contre-attaquer ne fut prise qu'il y a deux semaines, quand The Sun, le tabloïde sensationnaliste le plus lu du pays, transcrivit 23 minutes d'une soi-disant conversation téléphonique entre Diana et un ami la veille du Nouvel An 1989 (...) Elle se plaint de la «torture » qu'est son mariage et fixe un rendez-vous au mardi prochain, où elle sortirait sous prétexte d'aller chez un acuponteur » (VEJA, 9/9/1992, 1251, 38-39).

Comment les temps sont-ils discursivisés? La procédure de projection des temps du système énonciatif dans l'énoncé s'appellera débrayage temporel énonciatif. Le débrayage sera énoncif lorsque les temps du système énoncif s'établissent dans l'énoncé (Greimas e Courtés, 1979, 81). Le débrayage temporel peut se faire au premier ou au second degré. C'est le premier cas qui se produit lorsque les temps correspondent à la parole du narrateur ; le second lorsqu'ils résultent d'une délégation de voix opérée par le narrateur et sont de la sorte reliés au je interlocuteur. Le discours direct est donc caractérisé par le fait de contenir un débrayage de second degré. Dans les textes qui contiennent des discours directs, il y a un débrayage de premier degré qui installe un narrateur dans l'énoncé, et celui-ci, à son tour, réalise un nouveau débrayage, déléguant la voix à un personnage qui est ainsi instauré comme interlocuteur. Comme dans le discours direct il y a deux actes d'énonciation énoncés, en ce qui concerne la temporalisation, il y a deux moments distincts de référence, soit énonciatifs soit énoncifs. Du point de vue de l'organisation temporelle, nous avons deux moments, même lorsque le narrateur s'instaure comme interlocuteur, en se donnant la voix à lui-même. Si nous avons deux moments de référence, les temps de chaque énonciation s'organisent selon le moment de référence auquel ils se rapportent. Dans le discours indirect, il n'y a pas de débrayage de second degré et par conséquent, dans le passage du discours direct au discours indirect, le changement de deux moments de référence à un seul peut entraîner des transformations dans les marques temporelles, puisque celles-ci doivent parfois (et non toujours comme semblent le suggérer nos grammaires) changer du système énonciatif à l'énoncif. Dans ce passage, seuls sont altérés les temps du système énonciatif (présent, prétérit parfait 1 et futur du prétérit), quand le moment de référence du discours citant et du discours cité ne sont pas identiques. Dans ce cas, les temps du système énonciatif se transforment en temps correspondants du subsystème énoncif utilisé dans le discours citant (par exemple, le prétérit parfait 1 devient plus-que-parfait ou futur antérieur, si l'on emploie respectivement le subsystème passé ou futur).

64 À l'inverse du débrayage, qui est la projection hors de l'instance de l'énonciation des temps qui servent à constituer l'énoncé, soit un énoncé qui soit un simulacre de l'énonciation, soit un énoncé qui ne représente pas l'énonciation, l'embrayage temporel est l'effet de retour à l'instance de l'énonciation, produit par la suspension de l'opposition 
entre certains termes de la catégorie du temps (Greimas et Courtès, 1979, 119). En fait, le débrayage crée une énonciation énoncée où les temps de l'énoncé simulent les temps de l'énonciation ou un énoncé énoncé où l'on a l'illusion d'être devant la temporalité des évènements. On a l'impression de se trouver toujours en présence d'une temporalité non linguistique : temps de l'acte de dire, dans le premier cas; temps des évènements dans le second. Or, quand on neutralise des termes de la catégorie du temps, l'effet de sens obtenu est que le temps est une pure construction de l'énonciateur, qui présentifie le passé, rend présent le futur, etc. Ainsi, avec cette procédure, passe-t-on de l'illusion énonciative de la naturalité des temps du dire et du dit, de la chimère selon laquelle le temps linguistique est le temps du monde, à la certitude de ce que le temps est un effet de sens produit dans et par l'énonciation.

Dans le discours indirect libre, se mêlent deux voix: celle du narrateur et celle du personnage. Cependant, il n'y a pas de délégation de voix comme dans le discours direct, ni de lien entre la parole du personnage et l'énonciation du narrateur au moyen d'un verbum dicendi comme dans le discours indirect. Ce qui se produit c'est, comme pour la catégorie de personne, un embrayage des temps verbaux de la parole du personnage. On emploie, par conséquent, les temps verbaux du discours indirect avec la valeur de temps du discours direct.

Dans le cas des temps verbaux, on peut neutraliser :

1. un temps énonciatif et un énoncif correspondant;

2. un terme de la catégorie topologique, et un autre à l'intérieur du même système ou subsystème temporel ;

3. un terme de la catégorie topologique avec un autre d'un système ou subsystème temporel disctinct.

67 Nous nous trouvons devant le premier cas, quand se neutralisent, par exemple, le prétérit parfait 2 (concomitance limitée à un moment de référence passé) et le présent (concomitance à un moment de référence présent) réalisant ce qu'on appelle le présent historique, ou bien le présent et le prétérit imparfait, au profit du second temps du couple :

« En 1858, la Compagnie des Indes est supprimée, l'Empire Mongol également, et une vice-royauté est instaurée » (Delta, VIII, article Inde).

« Agora eu era o herói

E o meu cavalo só falava inglês

A noiva do cowboy era você,

Além das outras três.

Eu enfrentava os batalhões,

Os alemães e seus canhões.

Guardava o meu bodoque

E ensaiava o rock para as matinês ».

(Chico Buarque de Holanda)

Maintenant j'étais un héros

Et mon cheval ne parlait que l'anglais

La fiancée du cow-boy c'était toi,

Sans compter les trois autres.

Je faisais face aux bataillons,

Aux allemands avec leurs canons.

Je rangeais mon lance-pierre

Et je répétais le rock pour aller aux matinées. » 
prétérits imparfaits expriment une concomitance, non par rapport à une marque temporelle passée, mais au maintenant. Ces imparfaits appartiennent à l'indicatif, mode employé par le locuteur pour situer l'action dans la réalité. Toutefois, comme l'imparfait exprime l'imperfectif, c'est-à-dire qu'il présente le procès sans préciser ses limites initiale et finale, la langue l'emploie avec la valeur de présent pour créer un effet de sens d'irréalité, à savoir pour manifester de faits hypothétiques. De plus, comme l'imparfait situe les faits dans le passé, cet embrayage emploie le temps qui détache les faits du présent pour exprimer les événements détachés de la réalité (cf. Chevalier et alii, 1964, 343).

Analysons encore un exemple : futur antérieur à la place du prétérit plus-que-parfait :

« Desta (preocupação) é fácil possuirmo-nos, e nesta terei eu caído mais de uma vez ».

« Par celle-ci (cette préoccupation) il est facile d'être possédé et j'y aurai cédé (sans doute) plus d'une fois » (Herculano, apud Dias, 1970, 194).

Céder est antérieur à la marque temporelle passée plus d'une fois dans ma vie antérieure à ce moment-ci. Il est exprimé au futur antérieur pour teinter de doute la phrase.

Nous nous trouvons devant la seconde possibilité, lorsqu'est suspendue, par exemple, l'opposition entre concomitance (présent) et postériorité (futur du présent) du système énonciatif ou les mêmes termes de la catégorie topologique (prétérit imparfait et futur du passé) du subsystème énoncif du prétérit.

«D'ici un mois je suis de retour. Je pars demain; je ne veux pas qu'elle se doute de mon départ » (MA, I, 650).

«Ai, palavras, ai, palavras.

que estranha potência a vossa

Perdão podíeis ter sido!

- sois madeira que se corta "

(CM, 492)

«Ah! paroles, ah! paroles, Quel étrange pouvoir que le vôtre

Pardon vous auriez pu être!

- vous êtes bois qu'on coupe"

Le poème compare deux temps : un alors, temps de la vie, des promesses de liberté et un maintenant, temps de la prison, de la torture, de la mort. La concomitance du prétérit est indiquée par l'imparfait íeis. Par rapport à ce moment, dans un temps postérieur, les promesses auraient pu se réaliser. Cette probabilité est exprimé en portugais par l'imparfait podíeis (rendu en français par le passé du conditionnel) et non par le futur du prétérit, pour créer un effet de sens de certitude. Il indique que ce qui était prévu pour le futur était inévitable. Cette certitude, pourtant, a été abattue par la brutalité de la répression portugaise et le présent de l'indicatif montre que la mort a pris la place de ce qui semblait certain pour qui prêchait l'indépendance de la colonie. Le prétérit imparfait ayant valeur de futur du prétérit exprime donc une conséquence d'un fait passé, considérée inévitable par l'énonciateur, mais qui ne s'est pas réalisée.

73 Il y a un cas qui mérite une discussion plus détaillée : présent à la place du prétérit parfait 1:

«À la fin du déjeuner, je prends congé et je prends ma voiture, laissant le couple d'amis au restaurant (...) Ce fut hier » (FE).

74 Le complément circonstanciel de temps à la fin du déjeuner marque une antériorité par rapport au moment de l'énonciation. Cette antériorité est présentée, non par le prétérit 
parfait 1, mais par le présent. L'événement antérieur est présentifié pour montrer qu'il y a une résonance au présent, qui pèse davantage que le passé de l'action. Cette neutralisation présent vs passé simple 1 engendre par conséquent une amplification du présent en direction du passé. Le présent est vu alors comme une continuité logique ou psychologique du passé. On pourrait penser que dans ce cas, on a en fait, un prétérit parfait 2. Néanmoins, la concordance des temps, qui se réalise quand le verbe de la subordonnée est au subjonctif, sert à montrer s'il s'agit d'un prétérit parfait 1 ou d'un prétérit parfait 2, car, lorsque le verbe de la proposition principale est au prétérit parfait 1 et que le verbe de la subordonnée indique l'antériorité par rapport à celui de la principale, on emploie dans la subordonnée le prétérit parfait du subjonctif; dans les mêmes conditions, si le verbe de la principale est au prétérit parfait 2 , on emploie dans la subordonné le prétérit plus-que-parfait du subjonctif.

Textuellement :

« Le Consulat de France à São Paulo nia qu'il ait sollicité l'ouverture d'une enquête contre les personnes qui envoyèrent des lettres de protestation contre les tests nucléaires français dans le Pacifique Sud » (ESP, 12/10/1995, A9).

«Le 11 septembre, le Consulat de France à São Paulo nia qu'il eût sollicité l'ouverture d'une enquête sur les personnes qui envoyèrent des lettres de protestation contre les tests nucléaires français dans le Pacifique Sud ».

Ce que le français traduira ainsi :

«Le Consulat de France à São Paulo a nié avoir sollicité l'ouverture d'une enquête sur les personnes qui ont envoyé des lettres de protestation contre les tests nucléaires français dans le Pacifique Sud ».

Nous allons donner un dernier exemple : prétérit plus-que-parfait à la place du futur du prétérit :

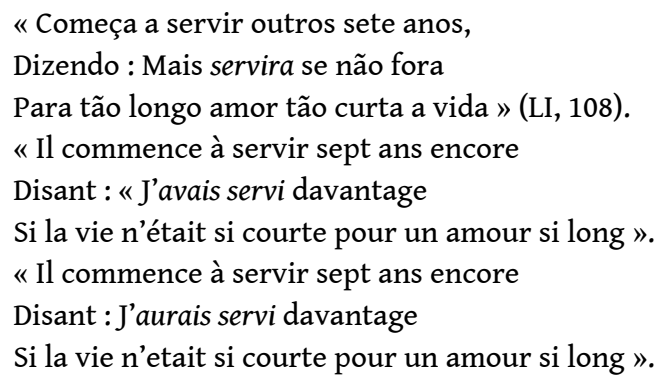

Le fait est narré au prétérit. Après le refus de Laban de donner à Jacob la main de Rachel, le berger commença (la concomitance par rapport au prétérit est exprimée ici par le présent) à servir Laban pendant une autre période de sept ans. Par rapport à son dire, qui est aussi au prétérit, l'action de servir est ultérieure. Cette postériorité n'est pas manifestée par le futur du prétérit, mais par le plus-que-parfait, pour créer un effet de sens d'inévitabilité de l'action future, étant donné que ce qui n'est même pas encore en train de se réaliser est montré comme quelque chose d'accompli.

Le troisième cas se produit, quand on neutralise, par exemple, la concomitance par rapport au moment de référence passé (présent) avec la postériorité à un moment de référence passé (futur du prétérit).

" Felizmente, ele reagiu depressa. Um passo a mais e o carro o esmaga».

«Heureusement, il réagit rapidement. Un pas de plus et la voiture l'écrase ».

Écrase indique la postériorité par rapport à la marque temporelle passée à ce moment-là. La finalité de cette construction est de souligner la fatalité de la conséquence par rapport à 
la cause exprimée. La conséquence est présentée comme réelle, bien qu'elle soit hypothétique.

Le résultat de la neutralisation se manifeste toujours au moyen de l'un des temps dont l'opposition a été suspendue. Il est clair que dans ce cas, un temps sera utilisé à la place d'un autre, car dans le cas contraire, la neutralisation ne serait pas sentie. On considérera un embrayage comme énonciatif, quand le résultat de la neutralisation est un temps énonciatif, ce qui se produit, soit quand on suspend les oppositions de temps du système énonciatif, soit quand on neutralise des temps des systèmes énonciatif et énoncif au bénéfice des premiers; on le classe comme énoncif, quand la neutralisation est manifestée par un temps énoncif, ce qui se produit, quand on neutralise des temps du système énoncif et énonciatif au profit des premiers, ou quand on suspend des oppositions entre des temps de chacun des systèmes énoncifs.

Ce qui a été dit à propos des temps verbaux s'applique aussi aux adverbes de temps. Il faut rappeler, cependant, qu'on ne neutralise que ceux qui manifestent effectivement le temps linguistique, et non les précisions chronologiques qu'on lui apporte. Par exemple, l'adverbe maintenant, qui indique une concomitance par rapport au moment de l'énonciation, peut être utilisé à la place de il y a peu de temps, tout à l'heure, et dans quelques instants, respectivement antériorité et postériorité par rapport au moment de l'énonciation, pour marquer un passé récent et un futur immédiat, à savoir, pour montrer la proximité du passé et du futur par rapport au moment de l'énonciation. En portugais on emploie un diminutif de agora, qui signifie maintenant (en somme, un petit maintenant), pour renforcer le caractère récent ou imminent, respectivement, du passé et du futur.

«Saiu inda agorinha » (PEM).

«Il est sorti il y a un instant ».

«Vamos começar a lição agorinha mesmo » (CR).

« Nous allons commencer la leçon tout de suite ».

«Estou fora dessa, cheguei agora ( = há pouco)».

" Je n'ai rien à voir avec ça, j'arrive à l'instant ».

83 Si l'on observe les neutralisations effectivement réalisées en portugais, on constate que la neutralisation n'a lieu que lorsqu'est en jeu un seul trait distinctif du système temporel (neutralisation de termes de la catégorie topologique dans le même système ou subsystème temporel, ou neutralisation de temps qui manifestent le même terme de la catégorie topologique dans des systèmes ou subsystèmes distincts); s'il y a deux traits à neutraliser (termes différents de la catégorie topologique de systèmes ou subsystèmes distincts), la neutralisation n'aura lieu que dans quelques situations particulières :

a) Quand on altère la situation énonciative. Par exemple, prétérit imparfait au lieu du prétérit parfait 1 :

«Trente-sept minutes de la première mi-temps étaient jouées' compléta le

folklorique Sílvio Luiz, de la chaîne Bandeirantes de télévision » (FSP, 13/6/1993, 5-5),

c'est-à-dire que :

"'Trente-sept minutes de la première partie du match s'étaient écoulées' compléta le

folklorique Sílvio Luiz, de la chaîne Bandeirantes de télévision ».

Les minutes du match déjà écoulées sont antérieures au moment de l'énonciation. Cette antériorité est exprimée, toutefois, par la concomitance durative par rapport au prétérit, ce qui établit une distance entre les instances de la narration et du narré. 
b) Quand il y a identité, du point de vue morphique, avec un temps admettant la neutralisation avec l'autre temps en jeu. Par exemple, futur du prétérit à la place du présent du futur :

"On se pose ici une question capable de faire perdre le sommeil à beaucoup de monde. Quand PC (Paulo Cesar Farias) se décidera à ouvrir la bouche, les institutions résisteraient-elles ? ( (FSP, 26/10/1993, 4-7).

Résister est concomitant avec la marque temporelle du futur quand PC se décidera à ouvrir la bouche. Cette concomitance est exprimée par le futur du prétérit (présent du conditionnel en français) pour marquer le doute de l'énonciateur concernant le fait. Cette neutralisation est identique, du point de vue morphique, à la neutralisation futur du prétérit et futur du présent.

88 c) Quand le présent est l'un des temps de l'opposition. Cet embrayage se produit sans doute, parce que le présent est parmi les temps celui qui a le plus de plasticité, étant donné qu'il indique présent, passé, futur, ponctualité, durativité, atemporalité. Par exemple, futur du prétérit 1 à la place du présent, qui a lieu, entre autres, quand on prétend transmettre une information non confirmée sur un évènement en cours, ou une information mise en doute par l'énonciateur.

«Pour le front, les russes se partagent en 'atlantistes' et 'euroasiatiques'. Les premiers défendraient le rapprochement de la Russie avec l'Occident et seraient des traitres des «traditions». Les militants du front, qui se jugent 'euroasiatiques' mêlent l'adoration de Staline à celle du tzar Nicolas II » (FSP, 25/9/1993, 2-14).

Une question se pose alors : pourquoi peut-on dire, dans un cas qu'il y a une erreur dans l'emploi des marques de temporalité, et que dans l'autre il y a un embrayage? Par exemple, dans le premier des textes suivants, nous pourrions dire qu'il y a une erreur; dans le second, un embrayage. Qu'est-ce qui les distingue?

a) «Le mardi 13, j'ai assité à une seule séance, dont les communications ont porté sur la morphosyntaxe; à 9 heures, Características formales y funcionales del sintagma en el sistema de la lengua española, de M. Dominguez, d'Espagne; la communication prochaine ( = suivante) n'a pas eu lieu en raison de l'absence du chercheur » (X, Rapport Scientifique adressé à la FAPESP, 24/4/1993, 13).

b) « Viens dîner tout à l'heure avec moi à Santa Tereza, dit Palha en prenant congé. Pas d'hésitation, je vous attends là-bas » ( = je vous attendrai) (MA, I, XXIII).

Il convient tout d'abord de placer ici de plus amples considérations sur la violation des paramètres grammaticaux. La transgression peut être commise par erreur ou intentionnellement. Dans le second cas, enfreindre les règles vise à produire un certain effet de sens; dans le premier l'infraction ne produit pas d'effet de sens, mais donne l'impression que quelque chose dans le texte n'est pas à sa place. En effet, dans le premier texte donné en exemple, rien ne justifie l'emploi de prochaine pour suivante. L'énonciateur se servait du système énoncif, indiqué par la marque temporelle passée mardi 13, et le passage du système énonciatif ne produit aucun effet de sens. Au contraire, dans le second texte, l'emploi du présent à la place du futur du présent montre que la présence de l'interlocutaire à Santa Tereza n'était pas envisagée par l'interlocuteur comme une chose possible mais comme certaine.

91 La question se présente maintenant de savoir comment on reconnait l'embrayage temporel au fil du discours. Si, comme le disent Greimas et Courtès, l'embrayage présuppose un débrayage antérieur, vu qu'il n'existe pas de neutralisation sans qu'il y ait une opposition, «l'embrayeur doit laisser quelque marque discursive du débrayage antérieur» (1979, 119). Lorsqu'a lieu un embrayage temporel, il doit y avoir, par 
conséquent, dans le contexte une marque temporelle permettant de dire qu'un temps verbal ou un adverbe sont employés avec la valeur d'un autre. Par exemple, dans D'ici un mois je suis de retour. Je pars demain (MA, I, VIII), on constate que le présent a la valeur de futur du présent et qu'il y a donc un embrayage, parce que d'ici un mois et demain indiquent que l'événement exprimé par le verbe est postérieur au moment de l'énonciation.

Le système de temps s'organise dans la langue d'une façon absolument symétrique. Néanmoins, le discours en mélangeant des perspectives, en confondant les termes de la catégorie topologique, en subvertissant les oppositions, produit un "vertige temporel » dont il se sert pour créer des effets de sens. Ces derniers ne se fragmentent pas en une multiplicité incontrolable de significations, mais sont subordonnés à la catégorie sémantique : approximation vs éloignement

À un premier niveau de concrétisation, cette catégorie en produit trois autres :

\begin{tabular}{|l|l|l|}
\hline \multicolumn{2}{|l|}{$\mid \begin{array}{l}|l| \\
\text { - une pour les effets de sens temporels stricto sensu : }\end{array}$} \\
\hline \hline & conjonction vs déplacement ; \\
\hline & - une autre pour les effets de sens modaux : \\
\hline & réalité vs virtualité ; \\
\hline & - une autre encore pour les effets de sens aspectuels : & \\
\hline & inaccompli vs non commencé. \\
\hline
\end{tabular}

De cette façon, nous avons un effet de sens de base pour chacune des catégories qui ont une incidence sur l'événement exprimé par le verbe.

Si nous considérons conjonction, réalité, inaccompli comme le terme A d'une opposition sémantique et déplacement, virtualité, non commencé comme le terme B et que nous appliquions une opération de négation à cette catégorie, nous obtiendrons non-conjonction , non-réalité et accompli comme terme non-A et non-déplacement, non-virtualité et accompli comme terme non-B. La concomitance exprimera des effets de sens contenus dans le lieu A ; la postériorité, ceux qui sont contenus dans le lieu B ; l'antériorité ceux du lieu non-B, quand elle est neutralisée par un temps de postériorité, et ceux du lieu non-A quand elle est à la place d'une concomitance.

Les effets de sens opèrent sur deux niveaux :

1. à l'intérieur d'un même subsystème, quand on emploie, par exemple, une postériorité avec valeur de concomitance.

2. dans des subsystèmes distincts (ainsi, l'utilisation du futur du prétérit avec la valeur de futur du présent - le présent du conditionnel pour le futur de l'indicatif - est le passage de la concomitance, puisque le futur appartient au système qui a pour moment de référence le moment de l'énonciation, pour l'antériorité, le futur du prétérit - conditionnel présent appartenant au subsystème dont le moment de référence est passé ; pour cette raison, cet embrayage produit un effet de sens d'irréalité). 
la détermination des effets de sens, le moment de référence prédomine sur les termes de la catégorie topologique. La conjonction peut se concrétiser comme résonance au moment de référence; le déplacement, comme une séparation totale du moment de référence; le non-déplacement, comme proximité, et la non-conjonction, comme éloignement.

«Il était venu tout jeune du Zaïre, il y était né. Ses parents s'étaient exilés au Zaire pendant la guerre d'indépendance de l'Angola et il naquit à Kinshasa. Il revint avec l'indépendance, apprit le portugais, oublia le lingala et s'intégra » (L, 122).

Le roman Lueji, de Pepetela est narré au prétérit. Par rapport à un moment de référence (année 1999), la venue au Zaïre. La naissance du personnage et l'exil de ses parents sont antérieurs et pour cette raison, sont narrés au prétérit plus-que-parfait. Cependant sont également antérieurs son retour, l'apprentissage du portugais, l'oubli du lingala et l'intégration. Pourtant ils sont relatés au prétérit parfait 2 (concomitance ponctuelle par rapport au moment de référence), parce que ces faits ont une résonance au moment du récit, car, comme l'explique le narrateur, les « anciens émigrés » de retour n'étaient pas bien vus en Angola depuis l'indépendance.

a réalité peut se manifester comme certitude relativement aux actions futures, comme inévitabilité, comme nécessité; la virtualité, comme supposition, doute, hypothèse, incertitude, probabilité, atténuation des faits; la non-virtualité, comme inéluctabilité de l'action future; la non-réalité, comme atténuation de l'action présente et évasion de la réalité.

«- Ele não quer que eu fique solteira a vida toda, não?

- Não tem poder para isso. Mas bem que queria, acho, ou não sabes, maninha? » (L, 136).

«- Il ne veut tout de même pas que je reste veille fille, non?

- Il n'en a pas le pouvoir. Mais il voudrait bien, je crois. Est-ce que tu ne le sais pas, soeurette?».

Le dialogue est ancré au moment de référence présent. Au lieu de dire Mais je crois qu'il le souhaite, qui indique la réalité, on dit, Mais il voudrait bien, je crois, qui signale la nonréalité, qui se manifeste comme atténuation, puisque il voudrait (dans le texte en portugais il est dit voulait) est un temps du subsystème d'antériorité.

101 L'inaccompli manifeste l'inévitabilité de l'action future, parce qu'il la montre comme déjà commencée, et l'imperfectivité dans l'événement passé; le non-commencé, le caractère éventuel de l'événement; le commencé, l'imminence de l'événement futur et la perfectivité de la résolution prise ; l'accompli, l'effet inéluctable de ce qui est en train de se produire, et la rapidité avec laquelle cela se produit.

«- C'est Ndumba uo Tembo que vous devez remercier, c'est lui le grand chasseur qui a tout fait.

Non seulement elle était courageuse comme peu; mais elle était modeste comme doit l'être un élu des esprits, disaient les anciens, et les plus jeunes approuvaient. Ndumba faisait un sourire forcé, car la victoire lui échappait » (L, 134, 5).

Tout le peuple faisait fête à la reine parce qu'elle avait soi-disant tué le lion qui allait dévorer Ndumba uo Tembo. En reálité, c'était lui qui avait tué le lion. Ainsi, lorsque le peuple commence à louer la reine, la victoire lui a déjà échappé. En utilisant l'imparfait (concomitance durative) au lieu du prétérit plus-que-parfait, le narrateur crée un effet de sens d'imperfectivité du fait passé, d'un long déroulement qui signale que tout blessait lentement l'amour propre du guerrier. 
Les temps, dans le discours, échappent aux conventions rigides du système, se mêlent, se superposent, se poursuivent, servent de contrepoint les uns aux autres, s'éloignent, se rapprochent, se combinent, se succèdent dans un jeu enchevêtré d'articulations et d'effets de sens. Cependant, dans ce contrepoint, ils obéissent à des règles, à des contraintes sémantiques. Le discours crée le cosmos et déteste le chaos.

En travaillant la temporalité dans le texte, il faut aussi distinguer le temps de la narration et le temps du narré. Le temps de la narration est toujours le présent, qui est le moment où l'on parle. Il est postérieur à l'histoire racontée, qui lui est donc antérieure. Si le prétérit est le temps par excellence du narré, le narrateur peut également créer une narration où il $\mathrm{y}$ ait concomitance entre les temps de la narration et de ce qui est narré (récits au présent), et une autre, appelée récit prophétique, dans lequel le temps de ce qui est narré est considéré postérieur à celui de la narration. Il y a de la sorte trois relations entre le temps de la narration et celui du narré. Celui-ci peut être concomitant, antérieur ou postérieur à celui-là. D'autre part, comme relativement à chaque moment de référence il y a une concomitance, une antériorité et une postériorité, les temps de la narration, au cas où cette instance est narrativisée, sont le présent, le prétérit parfait 1 et le futur du présent. De leur côté, les temps du narré sont le présent, le prétérit parfait 1 et le futur du présent dans les récits où il $\mathrm{y}$ a concomitance entre narration et narré ; prétérit parfait 2 , prétérit imparfait, prétérit plus-que-parfait et futur du prétérit simple et composé (le conditionnel présent et passé en français), dans les récits où la narration est postérieure à ce qui est narré ; présent du futur, futur antérieur et futur du futur dans les récits où la narration est antérieure à ce qui est narré.

Nous avons parlé de l'embrayage en tant qu'utilisation d'un temps à la place d'un autre à l'intérieur du texte. Nous avons également un macro-embrayage, qui régit la relation entre l'instance de la narration et l'instance du narré. Prenons comme exemple le poème Profondément, de Manuel Bandeira :

Quand hier je me suis endormi

La nuit de la Saint-Jean

Il y avait joie et rumeur

Éclatement de pétards feux de Bengale

Voix chants et rires

Auprès des feux allumés.

Au milieu de la nuit je me suis éveillé

Je n'entendis plus de voix ni de rire

Seulement des ballons

Passaient errants

Silencieusement

De temps en temps à peine

Le bruit d'un tramway

Tranchait le silence

Comme un tunnel

Où étaient ceux qui tout à l'heure

Dansaient

Chantaient

Et riaient

Auprès des feux allumés ?

Ils dormaient tous

Ils étaient tous allongés

Dormant

Profondément

*** 
Je n'ai pas pu voir la fin de la fête de Saint-Jean

Quand j'avais six ans

Parce que je me suis endormi

Aujourd'hui je n'entends plus les voix de ce temps-là

Ma grand-mère

Mon grand-père

Tonton Rodrigues

Tomasia

Rosa

Où sont-il tous?.

Ils dorment tous

Ils sont tous couchés

Ils dorment

Profondément »

Quand nous arrivons à la deuxième partie du poème, nous comprenons qu'hier est la veille du jour de la Saint-Jean de l'année où le poète avait six ans (dans ce temps-là). Le narrateur, après avoir relaté la fête de Saint-Jean dans une perspective temporelle énonciative (quand hier je me suis endormi), la situe dans une temporalisation énoncive (quand j'avais six ans / Je n'ai pas pu voir...) et alors, hier devient la veille. L'antériorité par rapport au présent est transformée en concomitance avec la marque temporelle passée. Ainsi, l'énoncé relaté dans le système énonciatif se métamorphose en énoncé avec une temporalisation énoncive. L'énonciateur l'a rapproché à travers la mémoire, puis l'a éloigné, replaçant les événements évoqués dans la catégorie des faits passés.

Le discours, au moyen d'un jeu complexe entre les temporalités de la narration et du narré, entre simultanéités, antériorités et postériorités, crée un temps qui simule l'expérience temporelle de l'homme. Si le récit est un simulacre de l'action de l'homme dans le monde, sa temporalité est un simulation de l'expérience du temps, qui se constitue à partir du moment où un je prend la parole, où comme l'enseigne Saint Augustin dans le livre XI des Confessions, le présent est un passage, le passé est la mémoire et le futur est l'attente. La fugacité du temps est le support de la narrativité et de l'expérience vécue. Dans le texte nous pouvons contempler le temps, et comme le disait Simone Weil, « la contemplation du temps est la clef de la vie humaine ».

\section{BIBLIOGRAPHIE}

ACADEMIA REPUBLICII SOCIALISTE ROMÂNIA (1966) Gramatica limbii române. Bucareste. Editura Academiei Republicii Socialiste România, v. I.

Augustin, Saint (1989) Confessions, Paris, Les Belles Lettres, t. 2.

BAKHTIN, M. (1979) Marxismo e filosofia da linguagem, São Paulo, Hucitec.

BENVEnISTE, E. (1966) Problèmes de linguistique générale, Paris, Gallimard, vol. I.

(1974) Problèmes de linguistique générale, Paris, Gallimard, vol. II.

CHEVALIER, J.-C. et alii (1964) Grammaire Larousse du français contemporain, Paris, Larousse. 
DIAS, Augusto Epiphanio da Silva (1970) Sintaxe histórica portuguesa, Lisboa, Livraria Clássica Editora.

FIORIN, J. L. (1996) As astúcias da enunciação : as categorias de pessoa, espaço e tempo, São Paulo, Ática. GREIMAS, A. J. e COURTES, J. (1979) Sémiotique. Dictionnaire raisonné de la théorie du langage, Paris, Hachette, vol I.

Guillaume, G. (1968) Temps et verbe, Paris, Champion.

IMBS, P. (1968) L'emploi des temps verbaux en français moderne. Essai de grammaire descriptive, Paris, Klincksieck.

mateus, M. H. M. et alii (1983) Gramática da Língua Portuguesa, Coimbra, Almedina.

PARRET, H. (1988) Enunciação e pragmática, Campinas, Editora da UNICAMP.

SERIANNI, L. (1989) Grammatica italiana, Torino, Utet.

\section{Exemples}

CM MEIRELES, Cecília. Obra poética, Rio de Janeiro, Nova Aguilar, 1985.

CR HOMEM, J. Cabra das rocas, São Paulo, Ática, 1973.

DELTA Grande Enciclopédia Delta Larousse, Rio de Janeiro, Editora Delta, 1973.

ESP O Estado de S. Paulo, 12/10/1995.

FSP Folha de S. Paulo, 13/6/1993 ; 25/9/1993 ; 26/10/1993 ;

L PEPETELA. Lueji, Luanda, União de Escritores Angolanos, 1989.

LI CAMÕES, L. de. Lírica. 4 ed. São Paulo, Cultrix, 1972.

MA ASSIS, M. de. Obra completa. Rio de Janeiro, Nova Aguilar, 1979, v. I.

MB BANDEIRA, M. Poesia completa e prosa, Rio de Janeiro, Nova Aguilar, 1983.

PEM KUhNer, M. H. Pedro Malazarte, Revista do Teatro, 471, Rio de Janeiro, Marco, 1989.

T ALENCAR, J. de. Til, São Paulo, Saraiva, 1968.

VEJ VEJA, 9/9/1992, 1251; 30/12/1992, 1268; 10/10/1995.

VGP VeRíssimo, É. A volta do gato preto, Porto Alegre, Globo, 1947.

\section{NOTES}

1. Imbs conseillait, en critiquant la proposition de Damourette et Pichon, de distinguer en français un système nuncal (de nunc) et un système tuncal (de tunc), et il avançait, pour sa part qu'il y a deux systèmes temporels, celui du maintenant et celui de l'alors, ce dernier se subdivisant en un alors passé et un alors futur $(1968,176-177)$. Il faut noter, toutefois, qu'ensuite, dans la mise en ordre des temps, Imbs tient compte de la morphologie et non de la sémantique (1968, 183-189).

2. Peu importe que la simultanéité soit réelle ou non. La Linguistique n'opère pas avec le monde « réel » mais avec le monde du langage et, par conséquent, avec des effets de sens.

3. Ce cas représente par rapport au moment de référence présent ce que le prétérit parfait représente dans le subsystème temporel du préterit. 
4. Cet emploi représente dans le système temporel présent ce que l'imparfait représente dans le subsystème du prétérit.

\section{RÉSUMÉS}

A partir des études de Benveniste sur l'énonciation, on observe qu'il existe dans la langue deux systèmes temporels: l'un directement lié au moment de l'énonciation, l'autre ordonné en fonction de moments de référence installés dans l'énoncé et situés, l'un dans le passé, l'autre dans le futur par rapport au moment d'énonciation. De la sorte nous avons un système énonciatif dans le premier cas et un système énoncif, avec deux subsystèmes, dans le second. Le moment d'un événement quelconque est déterminé par rapport aux différents moments de référence, et ce rapport est lui-même est défini par l'application de la catégorie topologique concomitance vs nonconcomitance (antériorité vs postériorité) aux différents moments de référence. Il y a donc trois moments structurellement remarquables dans la constitution d'un système temporel : le moment de l'énonciation (ME), le moment de la référence (MR) et le moment de l'événement (MA).De sorte que si nous tenons compte, non de la morphologie de la langue mais des relations constitutives de la catégorie du temps, nous avons neuf temps dans la langue. Le système temporel s'organise dans la langue de façon absolument symétrique. Néanmoins le discours, en multipliant les perspectives, en confondant les termes de la catégorie topologique, en subvertissant les oppositions, produit un « vertige temporel » dont il se sert pour créer des effets de sens. Cependant dans ce contrepoint ils obéissent à des règles, à des contraintes sémantiques. Le présent travail décrit le système temporel de la langue et étudie les procédures de discursivisation du temps dans le discours, les effets de sens relevant des différentes procédures de l'énonciation du temps et d'une manière générale les règles et les contraintes sémantiques auxquelles obéit la temporalisation discursive.

Starting from Benveniste's studies on utterance (énonciation) one notices the existence of two temporal systems in language: one "enunciative" (énonciatif), related to the moment of utterance; the other " enuncive " (énoncif) related to two moments of utterance installed in the utterance and situated in the past or in the future, regarding the moment of utterance. The moment of any given event is determined by the relationship of concomitance or nonconcomitance (anteriority or posteriority) it holds with the different moments of reference. Therefore, there are three structurally observable moments in the constitution of the temporal system- the moment of enunciation; the moment of reference and the moment of event; from whose relationship nine tenses result.

The temporal sytem follows a completely symmetric organization in language. As far as discourse is concerned it produces by multiplying perspectives, by mixing the terms of topological category, by subverting oppositions, a "temporal vertigo " of which discourse makes use to create meaning effects deriving from the different procedures of enunciation of time and, on the whole, from the rules and the semantic constraints the discoursive temporalization obeys. 
INDEX

Mots-clés : temporalisation, système énonciatif, système énoncif, débrayage temporel, embrayage temporel

Keywords : temporalization, enunciative system, enuncive system, temporal shifting out, temporal shifting in.

\section{AUTEUR}

JOSÉ LUIZ FIORIN

Université de São Paulo 